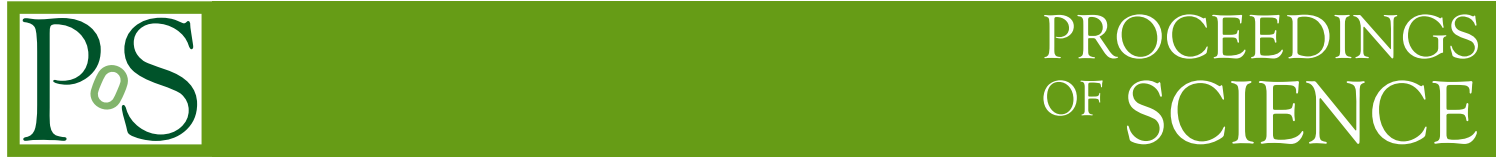

\title{
Experience From OPERA
}

\section{Antoine Cazes, on behalf of the OPERA collaboration*}

Laboratori Nazionali di Frascati - Via E. Fermi,40 - 00044 Frascati (Rome) Italy

E-mail: antoine.cazes@lnf.infn.it

The OPERA experiment aims at observing the $v_{\tau}$ appearance from the CNGS $v_{\mu}$ beam. The $v_{\tau}$ $\mathrm{CC}$ interactions are identified by reconstructing the $\tau$ decay topology using an Emulsion Cloud Chamber (ECC). OPERA is the first experiment to use emulsion at an industrial scale. It could therefore serve as a model for a future neutrino detector for the so called silver channel of a neutrino factory.

10th International Workshop on Neutrino Factories, Super beams and Beta beams

June 30 - July 52008

Valencia, Spain

${ }^{*}$ Speaker. 


\section{Introduction}

The golden channel studied with a neutrino factory is the observation of the $v_{\mu}$ appearance within the $v_{e}$ of the beam, but this measurement leaves unsolved the intrinsic degeneracy. An experiment able to measure the $v_{e} \rightarrow v_{\tau}$ oscillation would be able to solve this degeneracy, and this is called the silver channel of the neutrino factory [1].

The OPERA experiment [2] aims at discoving the $v_{\tau}$ appearance in the CNGS (Cern Neutrino to Gran Sasso) $v_{\mu}$ beam. $v_{\tau}$ are identified through their charged current interaction using the very precise reconstruction of the $\tau$ decay topology that is provided by the emulsion technology.

To accumulate the necessary statistics, the OPERA target needs to be at the kiloton scale. In the past, ECC was manufactured, therefore an important R\&D program has been carried out to produce them with industrial processes. This give to the OPERA collaboration a unique experience of a $v_{\tau}$ detection technique that can be adapted for the silver channel.

The OPERA experiment will be presented in this paper. First we describe the detectors, then we present the most important challenges of the experiment and in the last section, we conclude and summarize the expected results of OPERA.

\section{The OPERA detector}

OPERA has been built in the Hall C of the Gran Sasso underground laboratory, at $732 \mathrm{~km}$ from CERN. It stands bellow $1400 \mathrm{~m}$ of rock (which are equivalent to $3500 \mathrm{~m}$ of water ). It is an hybrid detector composed of a $1.35 \mathrm{kt}$ lead/emulsion target, and an electronic detector. OPERA is divided in two identical super-modules, each one made of one scintillator tracker, which contains also the target and one spectrometer. A series of automated microscopes located in the various laboratories of the collaboration complete the apparatus.

\subsection{The Emulsion Cloud Chamber}

The core of OPERA are the Emulsion Cloud Chambers (ECC) hereafter called bricks. They are basic units capable to detect a $v_{\tau}$ charged current interaction [3]. An ECC is a sandwich made of 56 lead plates, $1 \mathrm{~mm}$ thick, and 57 emulsion films, sizing $128 \times 102 \times 79 \mathrm{~mm}^{3}$, and weighting $8.3 \mathrm{~kg}$. The OPERA films have two emulsion layers ( $44 \mu \mathrm{m}$ thick) on a plastic base ( $205 \mu \mathrm{m}$ thick).

A $v_{\tau}$ that interacts by charged current inside a lead plate produces a $\tau$ that travels typically $1 \mathrm{~mm}$ before decaying in leptonic or semi-leptonic channels. Then, the very precise reconstruction of the event topology with the emulsions allows identifying the $v_{\tau}$ interaction. If the $\tau$ decays inside the lead plate where it as been produced, it will be identified by the impact parameter. Instead, if the $\tau$ traverses one or more emulsion films, it will be identified by the kink angle between the parent and the decay products. The background comes from the decay of charmed particles (since they decay with a comparable decay length, and in similar topological channels), the large angle scattering of muons and from hadron interactions.

On the downstream face of each brick, a separate box containing 2 emulsion films is attached. They will be scanned first in order to confirm the presence of the event predicted inside the brick.

The bricks are inserted inside walls $\left(\mathrm{L} \times \mathrm{H}=6.72 \times 7.33 \mathrm{~m}^{2}\right)$ in the detector. There are 31 walls per super-module. 


\subsection{The electronic detectors}

The ECC is a stand alone detector with no trigger. Electronic detectors are therefore required to trigger on an interaction and to locate the brick hit by a neutrino. The electronic detector is composed of one scintrillator tracker and one spectrometer per super-module.

\subsubsection{The Target Tracker}

The Target Tracker (TT) generates the trigger and performs the track reconstruction, the brick localization and a calorimetric measurement of the event shower.

There is one plane of vertical scintillator strips and one plane of horizontal strips behind each target wall. The strips are read out by wavelength shifting fiber connected at both side to a 64 channel Hamamatsu H7546 PMT. The scintillator strips are $6.86 \mathrm{~m}$ long and $1 \mathrm{~cm}$ thick, and they have a width of $2.5 \mathrm{~cm}$ [4]. The variation of the gain from channel to channel in such PMTs requires a read out chip with an adjustable gain for the pre-amplifier[5].

The alignment of the target component is an important point, knowing that for seismic safety, all the brick walls and TT planes are hang to the main structure. An additional effect comes from the deformation induced by the weight of the bricks ( $28 t /$ wall). For these reasons, measurements have been performed after the construction of the apparatus using a theodolite, in order to know the true geometry of the detector at the millimeter level.

The track reconstruction is performed with a cellular automaton method and the tracks are fitted using a Kalman filter.

\subsubsection{The spectrometers}

The OPERA spectrometers are built around dipolar magnets [6]. Each magnet consists of two arms, made of 12 slabs of iron (5 $\mathrm{cm}$ thick and $8.2 \mathrm{~m}$ high) connected by two return yokes surrounded by a coil in which flows $1600 \mathrm{~A}$.

Iron slabs are spaced by $2 \mathrm{~cm}$ gap housing RPC. They participate with the Target Tracker to the reconstruction of the track of the penetrating particles and to the calorimetric measurement of the hadronic shower[7]. The measurement of the deflection is provided by six groups of $8 \mathrm{~m}$ long drift tubes [8], that provide a spatial resolution of about $300 \mu \mathrm{m}$. Two groups of drift tubes are positioned in front of the spectrometer, two between the arms of the magnet, and two behind the spectrometer.

The OPERA spectrometer allows momentum measurement with a resolution of about $15 \%$ and a charge identification with an efficiency better than $99 \%$, up to $20 \mathrm{GeV}$ muons.

\subsection{The microscopes}

The emulsion films are read out by two types of automatic microscope, one developed in Japan and one in Europe[9]. The Japanese system is based on a very fast CCD camera read out by a dedicated electronic, while the European system is built using a CMOS camera and commercial components in a software-based framework. The microscopes scan the emulsion films on their entire surface, and by moving the focal distance, to measure the track at different depths. This allows reconstructing directly at the data taking level a 3D vector, with very high accuracy (about $2 \mu \mathrm{m}$ in position and $2 \mathrm{mrad}$ in angle). 


\section{The Construction Challenges}

They are 154750 bricks in the OPERA detector and one of the most important technical challenges of the experiment is to be able to handle them, from construction to analysis. Many points had to be addressed: production and insertion in OPERA, extraction and development of the brick hit by a neutrino, and scanning of these bricks. Therefore, this section may turns out to be instructive for future OPERA-like detectors for silver channel studies.

\subsection{The brick production}

The specifications of the bricks are very tight in order to fit them anywhere inside the detector, and a reasonable speed has to be maintained to produce the 150000 bricks of OPERA. A fully automated machine has been designed, called the Brick Assembly Machine (BAM).

The brick components have been selected with care. The lead was chosen with a low radioactivity $\left(<20 \alpha / \mathrm{cm}^{2} /\right.$ day and $<100 \beta / \mathrm{cm}^{2} /$ day), and a PbCa $(0.03 \%)$ alloy was employed because pure lead is too soft to be laminated and cut precisely.

8.8 millions of emulsion films have been produced for OPERA. A dedicated mass production chain has been built by the FUJI Company for the pouring of the gel on the plastic base, which was traditionally made by hand.

Emulsion is a delicate detector that has to be kept away from light and as much as possible from cosmic rays (they integrate the tracks of all charged particles by which they are traversed). For these reasons, the BAM has been installed inside one of the gallery of the underground laboratory in Gran Sasso, and part of the production chain is in a black room, which in addition must be a clean room, to avoid the deposition of dust inside the bricks.

The chain is composed of 5 groups of 2 robots with anthropomorphic arms. These robots are in charge of the piling (one for the lead, one for the emulsion). Once the pile is finished, the pressure is maintained by an aluminum structure, and at last, an anthropomorphic robot does the wrapping of the brick with an adhesive aluminum tape to grant light tightness. The last tasks of the BAM are to label the brick with bar codes and to add a box containing the CS. The main difficulties during the commissioning came from the lead stickiness, and from the bending of the emulsion films and their stickiness.

The production of all the OPERA brick took about one year and a half.

\subsection{The Brick Manipulating System}

Once the brick are built, they are inserted in the detector target walls by two dedicated robots, called the Brick Manipulating System (BMS). This system is also in charge of removing the bricks containing a neutrino interaction during the data tacking period. There is one robot along each side of the detector.

For insertion, the robot is placed in front of a row with the bricks. A drawbridge is used to transfer the bricks to the tray. They are inserted as a train, the last brick being pushed by a jack, and pushing the other inside the tray. For the extraction, a small vehicle equipped with a sucking grip is able to enter the detector and to remove the brick one by one until the one desired is reached. 


\section{Running of the experiment}

The automatisation of the experiment has been a permanent concern. Nevertheless, the running of OPERA requires an important man power. In addition of the running of a "usual" high energy physics electronic detector, OPERA has to handle about 30 bricks per day. The complete process to finaly obtain an event is the following: the brick is extracted and exposed to X-Ray to align the two emulsion films forming the CS with the brick. Then, the CS are removed, developed and scanned. If the CS scanning confirms the presence of an event inside the brick, the emulsion films inside the brick are marked again by X-Ray, and the brick is transported outside the underground laboratory, for a one day cosmic exposure for precise alignment. Finally, the brick is open and developed, and the emulsion films are sent to the scanning laboratories where they will be analysed. If the CS does not confirm the presence of the event, a new CS box is fixed to the brick, which is reinserted in the detector, and the process restart with the next probable predicted brick.

\section{Expected results and conclusion}

With the nominal intensity of the CNGS ( $4.510^{19}$ pot/year $)$, there will be $10.4 v_{\tau}$ observed in the OPERA detector in 5 years of data taking, and for $\Delta m_{23}^{2}=2.510^{-3} \mathrm{eV}^{2}$, and it can go up to 14.9 events if $\Delta m_{23}^{2}=310^{-3} \mathrm{eV}^{2}$. The backgroud will be of 0.7 event, that are due for one half to charm decay, and for the other half for large muon scattering or hadron interaction.

The OPERA experiment received its first beam for physics during a few weeks in 2007 and the first events have been scanned and analysed [10]. Since July 2008, the targets are filled, and OPERA is taking data with success. Bricks are extracted every day, they are developed and scanned in the various scanning laboratories. The OPERA collaboration already proved that it was possible to build a large emulsion detector, and is proving day after day that it is possible to handle it, justifing the choice made for the technology. Now physics is coming...

\section{References}

[1] A. Donini, D. Meloni and P. Migliozzi, Nucl. Phys. B 646 (2002) 321 [arXiv:hep-ph/0206034].

[2] M. Guler et al., An appearance experiment to search for $v_{\mu} \rightarrow v_{\tau}$ oscillation in the CNGS beam CERN/SPSC 2000-028, SPSC/P318, LNGS P25/2000, Jul. 2000.

[3] T. Nakamura et al., Nucl. Instr. Meth. A 556 (2006) 80.

[4] T. Adam et al., Nucl. Instr. Meth. A 577 (2007) 523.

[5] A. Lucotte et al., Nucl. Instrum. Meth. A 521 (2004) 378.

[6] M. Ambrosio et al, IEEE Trans. Nucl. Sci. 51 (2004) 975 ; A. Cazes et al, JINST 2 (2007) T03001.

[7] G. Di Iorio et al, Nucl. Phys. Proc. Suppl. 125 (2003) 27 ; M. Incurvati et al, Nucl. Instr. Meth. A 500 (2003) 441.

[8] R. Zimmermann et al, Nucl. Instr. Meth. A 555 (2005) 435.

[9] N. Armenise et al, Nucl. Instr. Meth. A 551 (2005) 261 ; L. Arrabito et al, Nucl. Instr. Meth. A 568 (2006) 578 ; L. Arrabito et al, JINST 2 (2007) P05004.

[10] R. Acquafredda et al, New J. Phys, 8 No12 (December 2006) 303 